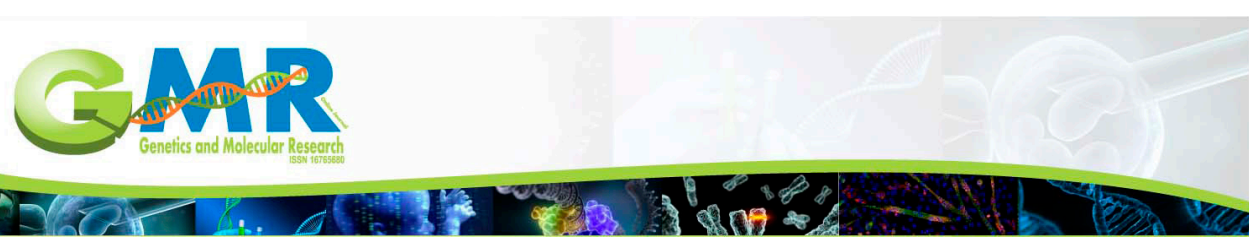

\title{
Prognostic role of microRNA-100 in patients with bladder cancer
}

\author{
Y.H. Cao, H.H. Zhang, H.F. Xu, Y.J. Duan, Q. Li and B. Huang \\ Department of Urology, First Affiliated Hospital of Nahua University, Hengyang, China \\ Corresponding author: Y.H. Cao \\ E-mail: nh_caoyouhan@126.com \\ Genet. Mol. Res. 14 (4): 15948-15954 (2015) \\ Received July 13, 2015 \\ Accepted October 7, 2015 \\ Published December 7, 2015 \\ DOI http://dx.doi.org/10.4238/2015.December.7.6
}

ABSTRACT. We investigated the clinical significance and prognostic value of microRNA-100 (miR-100) in bladder cancer. Quantitative realtime polymerase chain reaction was used to analyze the expression of miR-100 in 92 pairs of human bladder cancer and adjacent normal tissue samples. Overall survival (OS) curves were plotted using the Kaplan-Meier method and were evaluated for statistical significance using a log-rank test. The significance of different variables with respect to survival was analyzed using the multivariate Cox proportional hazard model. The miR100 expression level was significantly lower in bladder cancer tissues than in normal adjacent tissues (mean \pm SD: $1.49 \pm 0.52$ vs $2.79 \pm 0.59$, $\mathrm{P}<$ $0.05)$. A low miR-100 expression level was correlated with tumor stage ( $P$ $=0.023)$, tumor grade $(P=0.031)$, and regional lymph node involvement $(P=0.16)$. Kaplan-Meier analysis with log-rank test indicated that low miR100 expression had a significant impact on OS ( 35.1 vs $75.3 \%$; $P=0.004)$. Multivariate analysis revealed that the miR-100 expression level was an independent prognostic factor for OS $(\mathrm{HR}=2.768,95 \% \mathrm{Cl}=1.287-8.992$; $P=0.009)$ in bladder cancer patients. The present study demonstrated that the downregulation of miR-100 was associated with advanced clinical features and poor prognosis for bladder cancer patients, suggesting that 
miR-100 downregulation may be used as an unfavorable prognostic biomarker in bladder cancer.

Key words: Bladder cancer; MicroRNA-100; Prognosis; Biomarker

\section{INTRODUCTION}

Bladder cancer is the fourth most common cancer in men and the eighth leading cause of cancer-related deaths. An estimated 72,570 adults (54,610 men and 17,960 women) are diagnosed with bladder cancer in the United States annually (Siegel et al., 2014). Many established factors, such as smoking and exposure to toxins, are believed to contribute to tumorigenesis and progression of bladder cancer (Kiriluk et al., 2012). However, the exact molecular mechanisms underlying bladder tumorigenesis remain unclear (Volanis et al., 2011).

MicroRNAs (miRNAs) are small, single-stranded, non-coding RNAs that are highly conserved between species (Nelson et al., 2003). They are key post-transcriptional regulators of gene expression that act mainly by binding to the 3'-untranslated regions (3'-UTRs) of target mRNAs and thereby participate in many human biological processes, including growth, apoptosis, differentiation, and angiogenesis (Bartel, 2004; Berardi et al., 2012). In recent studies, dysregulation of miRNAs was found to play a critical role in tumorigenesis and tumor development (Jansson and Lund, 2012). Dysregulated expression of miR-100 has been found in various types of cancers. miR-100 can act as either an oncogene or a tumor suppressor in different cancers (Chen et al., 2014). Previously, Oliveira et al. (2011) found that miR-100 acted as a tumor suppressor in human bladder cancer. However, the clinical significance and prognostic value of miR-100 in bladder cancer have not been investigated.

\section{MATERIAL AND METHODS}

\section{Patients and specimens}

Our study was approved by the Ethics Committee of the First Affiliated Hospital of Nahua University. Informed consent was obtained from all patients. Ninety-two pairs of primary bladder cancer and adjacent normal bladder tissues were collected between April 2007 and July 2013 from the Department of Urology, the First Affiliated Hospital of Nahua University. The fresh tissue specimens were collected, immediately placed in liquid nitrogen, and then stored at $-80^{\circ} \mathrm{C}$ until required for the isolation of RNA. None of the patients had received preoperative chemotherapy or radiotherapy. Of the 92 patients, 27 underwent radical cystectomy, 26 underwent partial cystectomy, and 39 underwent transurethral resection of the bladder tumor. The duration of followup was calculated from the date of surgery to death or last follow-up, and patients were excluded if they had incomplete medical records or inadequate follow-up. All patients who died from diseases other than bladder cancer or from unexpected events were excluded from the case collection. The clinicopathologic characteristics of the patients with bladder cancer are shown in Table 1.

\section{TaqMan real-time quantitative reverse transcription polymerase chain reaction (qRT-PCR) for miRNA}

Total RNA was isolated from frozen specimens by homogenizing the tissue in TRIzol reagent (Invitrogen, Carlsbad, CA, USA), according to the manufacturer instructions. The purity and 
concentration of RNA were determined using a NanoDrop 1000 spectrophotometer (Thermo Scientific, Wilmington, DE, USA). The differentially expressed amount of miR-100 was validated in triplicate by qRT-PCR. Briefly, 2 mg RNA was added to the reverse transcription reaction, and the CDNA served as a template for amplification in the PCR with sequence-specific primers (Sangon Biotech, Shanghai, China) using a SYBR PrimeScript miRNA RT-PCR kit (TaKaRa Biotechnology Co. Ltd., Dalian, China) on a 7500 Real-Time PCR system (Applied Biosystems, Carlsbad, CA, USA). The PCR cycling regimen was as follows: denaturation at $95^{\circ} \mathrm{C}$ for 30 $\mathrm{s}$, followed by 40 cycles of annealing at $95^{\circ} \mathrm{C}$ for $5 \mathrm{~s}$, and extension at $60^{\circ} \mathrm{C}$ for $34 \mathrm{~s}$. Small nucleolar RNA U6 was used as an internal standard for normalization. The cycle threshold $\left(\mathrm{C}_{\mathrm{T}}\right)$ value was calculated. The $2^{-\Delta C T}\left(\Delta \mathrm{C}_{\mathrm{T}}=\mathrm{C}_{\text {TmiR-100 }}-\mathrm{C}_{\text {TUG RNA }}\right)$ method was used to quantify the relative amount of miR-100.

\section{Statistical analysis}

All statistical analyses were carried out using the SPSS 18.0 software package (SPSS, Chicago, IL, USA). The data are reported as means \pm SD. The Kruskal-Wallis test or the Mann-Whitney $U$ test was performed to compare microRNA levels between groups. The chisquare or Fisher exact probability test was used to examine possible correlations between miR-100 expression and clinical features. Overall survival (OS) curves were plotted using the Kaplan-Meier method and were evaluated for statistical significance using a log-rank test. The significance of different variables with respect to survival was analyzed using the multivariate Cox proportional hazard model. Differences were considered to be statistically significant when $P$ was less than 0.05 .

\section{RESULTS}

\section{miR-100 was significantly downregulated in bladder cancer tissues}

We analyzed the expression levels of miR-100 in 92 pairs of bladder cancer tissues and normal adjacent tissues from 92 patients with bladder cancer. As revealed by qRT-PCR analysis, the miR-100 expression level was significantly lower in bladder cancer tissues than in normal adjacent tissues (mean \pm SD: $1.49 \pm 0.52$ vs $2.79 \pm 0.59, P<0.05$, as shown in Figure 1).

\section{Reduced expression of miR-100 is associated with advanced clinicopathologic characteristics of patients with bladder cancer}

The 92 patients with bladder cancer were classified into two groups according to the median expression level of miR-100, as determined by qRT-PCR. Of the 92 patients with bladder cancer, 46 were placed in the low miR-100 expression group and 46 were placed in the high miR-100 expression group. The association between clinicopathologic features and miR-100 expression is summarized in Table 1. Low miR-100 expression level was correlated with tumor stage $(P=0.023)$, tumor grade $(P=0.031)$, and regional lymph node involvement $(P=0.16)$. However, low miR-100 expression was not associated with other clinicopathological factors of bladder cancer patients, including age, gender, and number of tumors (all $\mathrm{P}>0.05$, as shown in Table 1). 


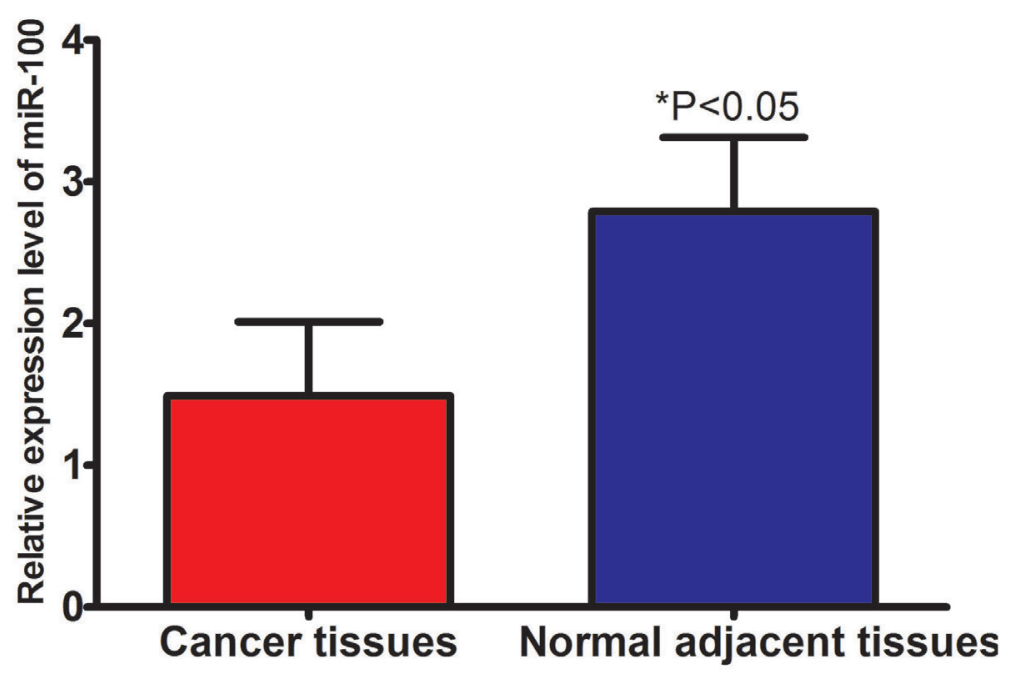

Figure 1. Expression of miR-100 determined by quantitative real-time PCR in 92 paired human bladder cancer and adjacent normal tissues. miR-100 expression levels were lower in tumor tissues than in adjacent normal tissues $(\mathrm{P}<$ 0.05).

\begin{tabular}{|c|c|c|c|c|}
\hline \multirow[t]{2}{*}{ Parameter } & \multirow[t]{2}{*}{ No. of cases } & \multicolumn{2}{|c|}{ miR-100 expression level } & \multirow[t]{2}{*}{$P$ value } \\
\hline & & Low $(N=46)$ & High $(N=46)$ & \\
\hline \multicolumn{5}{|l|}{ Age (years) } \\
\hline$<55$ & 43 & 19 & 24 & \multirow[t]{2}{*}{0.471} \\
\hline$\geq 55$ & 49 & 27 & 22 & \\
\hline \multicolumn{5}{|l|}{ Gender } \\
\hline Male & 57 & 27 & 30 & \multirow[t]{2}{*}{0.659} \\
\hline Female & 35 & 19 & 16 & \\
\hline \multicolumn{5}{|l|}{ Stage } \\
\hline Ta-T1 & 42 & 12 & 30 & \multirow[t]{2}{*}{0.023} \\
\hline$\geq T 2$ & 50 & 34 & 16 & \\
\hline \multicolumn{5}{|l|}{ Grade } \\
\hline $\mathrm{G} 1 / 2$ & 37 & 10 & 27 & \multirow[t]{2}{*}{0.031} \\
\hline G3 & 55 & 36 & 19 & \\
\hline \multicolumn{5}{|c|}{ Number of tumors } \\
\hline Single & 51 & 21 & 30 & \multirow[t]{2}{*}{0.071} \\
\hline Multiple & 41 & 25 & 16 & \\
\hline \multicolumn{5}{|c|}{ Regional lymph node involvement } \\
\hline Absent & 33 & 2 & 31 & \multirow[t]{2}{*}{0.016} \\
\hline Present & 59 & 44 & 15 & \\
\hline
\end{tabular}

\section{Association between miR-100 expression and survival in bladder cancer patients}

Kaplan-Meier analysis with the log-rank test indicated that low miR-100 expression had a significant impact on OS ( 35.1 vs $75.3 \% ; P=0.004$, as shown in Figure 2). Univariate and multivariate analyses were used to evaluate whether the miR-100 expression level and various clinicopathological features were independent prognostic parameters of bladder cancer patient outcomes. Multivariate analysis revealed that miR-100 expression level was an independent prognostic factor for OS (HR = 2.768, 95\% Cl: 1.287-8.992; $\mathrm{P}=0.009$ ) of bladder cancer patients (Table 2). 


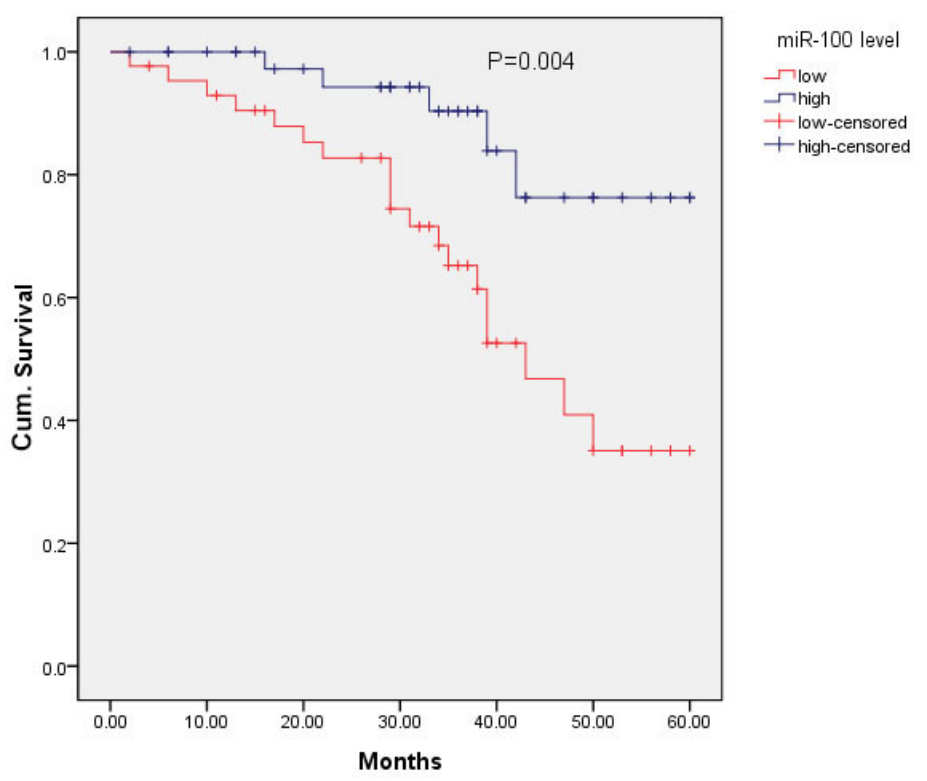

Figure 2. Kaplan-Meier survival curves of patients with bladder cancer on the basis of miR-100 expression status. Patients in the low expression group had significantly poorer prognosis than those in the high expression group $(\mathrm{P}=$ 0.004 , log-rank test).

\section{Table 2. Multivariate analyses for overall survival by Cox regression model.}

\begin{tabular}{|c|c|c|c|c|}
\hline Variable & Parameters & Hazard ratio & $95 \% \mathrm{Cl}$ & $P$ value \\
\hline Age (years) & $\geq 55$ vs $<55$ & 1.246 & $0.368-2.824$ & 0.561 \\
\hline Gender & Male vs female & 0.672 & $0.266-1.725$ & 0.788 \\
\hline Stage & $\geq \mathrm{T} 2$ vs $\mathrm{Ta}-\mathrm{T} 1$ & 3.165 & $1.572-10.812$ & 0.007 \\
\hline Grade & $\mathrm{G} 3$ vs G1/2 & 2.167 & $1.262-6.391$ & 0.016 \\
\hline Number of tumors & Multiple vs single & 2.081 & $0.572-3.198$ & 0.079 \\
\hline Regional lymph node involvement & Present vs absent & 1.879 & $1.028-7.265$ & 0.011 \\
\hline miR-100 expression level & Low vs high & 2.768 & $1.287-8.992$ & 0.009 \\
\hline
\end{tabular}

\section{DISCUSSION}

Bladder cancer is one of the leading causes of cancer-related deaths worldwide; therefore, finding new molecular targets for its diagnosis and treatment has the potential to improve the clinical therapy strategy and prognosis of the disease. MiRNAs are small endogenous non-coding RNAs that bind to partially complementary recognition sequences of target mRNAs, causing either degradation or preventing their translation (Nelson et al., 2003; Perera and Ray, 2007). Since their discovery in 1993, altered expressions of miRNAs have been associated with a variety of human diseases, including cancer (Perera and Ray, 2007; Subramanian and Steer, 2010; Roy and Sen, 2012). Identification of miRNA molecular profiles associated with the prognosis of patients with bladder cancer may not only elucidate the underlying biological mechanisms involved in the development or progression of the disease, but also provide the opportunity to identify novel targets for bladder cancer therapy. 
Dysregulated expression of miR-100 has been found in various types of cancers. miR-100 can act as either an oncogene or a tumor suppressor in different cancers (Chen et al., 2014). For example, Shi et al. (2010) reported that underexpressed miR-100 leads to Plk1 overexpression, which in turn contributes to nasopharyngeal cancer progression. Peng et al. (2012) demonstrated that low miR-100 expression may be an independent poor prognostic factor for human epithelial ovarian cancer. Huang et al. (2012) found that downregulation of miR-100 was significantly associated with advanced FIGO stage, the presence of lymph node metastasis, and reduced survival of small-cell carcinoma of the cervix. In addition to these, the downregulation of miR100 was also shown in hepatocellular carcinoma, oral cancer, and adrenocortical cancer (Henson et al., 2009; Doghman et al., 2010; Chen et al., 2013). In contrast, an upregulation of miR-100 has been observed in renal cell carcinoma, acute myeloid leukemia, and prostate cancer (Akbari Moqadam et al., 2013; Wang et al., 2013; Wang et al., 2014). Previously, Oliveira et al. (2011) found that miR-100 acted as a tumor suppressor in human bladder carcinoma 5637 cells. Their study demonstrated the effects of miR-100 on cell growth rates and clonogenicity capacity in the 5637 cell line, emphasizing a possible effect of this miRNA on bladder carcinoma pathogenesis. $\mathrm{Xu}$ et al. (2013) found that the mTOR gene was a direct target of miR-100. siRNA-mediated mTOR knockdown phenocopied the effect of miR-100 in bladder cancer cell lines. In addition, the cancerous metastatic nude mouse model established on the basis of primary bladder cancer cell lines suggested that miR-100/mTOR-regulated cell motility was associated with tumor metastasis. Both mTOR and p70S6K (downstream messenger) presented higher expression levels in distant metastatic foci such as in liver and kidney metastases than in the primary tumor. Taken together, miR-100 may act as a tumor suppressor in bladder cancer, and reintroduction of this mature miRNA into tumor tissue may prove to be a therapeutic strategy by reducing the expression of target genes (Xu et al., 2013). However, the clinical significance and prognostic value of miR-100 in bladder cancer have not been investigated. In the present study, we analyzed the expression levels of miR100 in 92 pairs of bladder cancer tissues and normal adjacent tissues from 92 patients with bladder cancer. As revealed by quantitative RT-PCR analysis, miR-100 expression level was significantly lower in bladder cancer tissues than in normal adjacent tissues. Low miR-100 expression level was correlated with tumor stage, tumor grade, and regional lymph node involvement. These results showed that decreased expression of miR-100 in bladder cancer was associated with significantly aggressive pathologic features, indicating that miR-100 might inhibit bladder cancer progression. Kaplan-Meier analysis with the log-rank test indicated that low miR-100 expression had a significant impact on OS. Univariate and multivariate analyses were used to evaluate whether the miR-100 expression level and various clinicopathological features were independent prognostic parameters of bladder cancer patient outcomes. Multivariate Cox proportional hazard model analysis showed that miR-100 expression was independently associated with the OS of patients with bladder cancer, indicating that miR-100 could be an independent prognostic factor of OS for patients with bladder cancer.

In conclusion, the present study demonstrated that the downregulation of miR-100 was associated with advanced clinical features and poor prognosis of bladder cancer patients, suggesting that miR-100 downregulation may be used as an unfavorable prognostic biomarker in bladder cancer.

\section{Conflicts of interest}

The authors declare no conflict of interest. 


\section{REFERENCES}

Akbari Moqadam F, Lange-Turenhout EA, Ariës IM, Pieters R, et al. (2013). MiR-125b, miR-100 and miR-99a co-regulate vincristine resistance in childhood acute lymphoblastic leukemia. Leuk. Res. 37: 1315-1321.

Bartel DP (2004). MicroRNAs: genomics, biogenesis, mechanism, and function. Cell 116: 281-297.

Berardi E, Pues M, Thorrez L, Sampaolesi M, et al. (2012). miRNAs in ESC differentiation. Am. J. Phys. Heart Circ. Physiol. 303: H931-H939.

Chen $P$, Zhao $X$ and Ma $L$ (2013). Downregulation of microRNA-100 correlates with tumor progression and poor prognosis in hepatocellular carcinoma. Mol. Cell. Biochem. 383: 49-58.

Chen $\mathrm{P}, \mathrm{Xi} \mathrm{Q}$, Wang $\mathrm{Q}$ and Wei $\mathrm{P}$ (2014). Downregulation of microRNA-100 correlates with tumor progression and poor prognosis in colorectal cancer. Med. Oncol. 31: 235.

Doghman M, El Wakil A, Cardinaud B, Thomas E, et al. (2010). Regulation of insulin-like growth factor-mammalian target of rapamycin signaling by microRNA in childhood adrenocortical tumors. Cancer Res. 70: 4666-4675.

Henson BJ, Bhattacharjee S, O'Dee DM, Feingold E, et al. (2009). Decreased expression of miR-125b and miR-100 in oral cancer cells contributes to malignancy. Genes Chromosome.Canc. 48: 569-582.

Huang L, Lin JX, Yu YH, Zhang MY, et al. (2012). Downregulation of six microRNAs is associated with advanced stage, lymph node metastasis and poor prognosis in small cell carcinoma of the cervix. PloS One 7: e33762.

Jansson MD and Lund AH (2012). MicroRNA and cancer. Mol. Oncol. 6: 590-610.

Kiriluk KJ, Prasad SM, Patel AR, Steinberg GD, et al. (2012). Bladder cancer risk from occupational and environmental exposures. Urolo. Oncol. 30: 199-211.

Nelson P, Kiriakidou M, Sharma A, Maniataki E, et al. (2003). The microRNA world: small is mighty. Trends Biochem. Sci. 28: 534-540.

Oliveira JC, Brassesco MS, Morales AG, Pezuk JA, et al. (2011). MicroRNA-100 acts as a tumor suppressor in human bladder carcinoma 5637 cells. Asian Pac. J. Cancer Prev. 12: 3001-3004.

Peng DX, Luo M, Qiu LW, He YL, et al. (2012). Prognostic implications of microRNA-100 and its functional roles in human epithelial ovarian cancer. Oncol. Rep. 27: 1238-1244.

Perera RJ and Ray A (2007). MicroRNAs in the search for understanding human diseases. BioDrugs 21: 97-104.

Roy S and Sen CK (2012). miRNA in wound inflammation and angiogenesis. Microcirculation 19: 224-232.

Shi W, Alajez NM, Bastianutto C, Hui AB, et al. (2010). Significance of PIk1 regulation by miR-100 in human nasopharyngeal cancer. Int. J. Cancer 126: 2036-2048.

Siegel R, Ma J, Zou Z and Jemal A (2014). Cancer statistics, 2014. CA Cancer J. Clin. 64: 9-29.

Subramanian S and Steer CJ (2010). MicroRNAs as gatekeepers of apoptosis. J. Cell Physiol. 223: 289-298.

Volanis D, Papadopoulos G, Doumas K, Gkialas I, et al. (2011). Molecular mechanisms in urinary bladder carcinogenesis. J. BUON 16: 589-601.

Wang G, Chen L, Meng J, Chen M, et al. (2013). Overexpression of microRNA-100 predicts an unfavorable prognosis in renal cell carcinoma. Int. Urol. Nephrol. 45: 373-379.

Wang M, Ren D, Guo W, Wang Z, et al. (2014). Loss of miR-100 enhances migration, invasion, epithelial-mesenchymal transition and stemness properties in prostate cancer cells through targeting Argonaute 2. Int. J. Oncol. 45: 362-372.

Xu C, Zeng Q, Xu W, Jiao L, et al. (2013). miRNA-100 inhibits human bladder urothelial carcinogenesis by directly targeting mTOR. Mol. Cancer Ther. 12: 207-219. 